\title{
FINDING CENTRE OF GRAVITY OF POSTURE SEQUENCES OF ASANAS USING SKELETAL INFORMATION AS JOINT ANGLE DIFFERENCES
}

\author{
PONMOZHI CHEZHIYAN ${ }^{1}$, DR. P. DEEPA LAKSHMI ${ }^{2} \&$ DR. ANISHA $M^{3}$
}

${ }^{1}$ Assistant Professor, Department of Computer Applications, Kalasalingam Academy of Research and Education, Viruthunagar, Tamil Nadu, India

${ }^{2}$ Professor, Department of Computer Science and Engineering, Kalasalingam Academy of Research and Education, Viruthunagar, Tamil Nadu, India

${ }^{3}$ Assistant Professor, Department of Biomedical Engineering, Kalasalingam Academy of Research and Education, Viruthunagar, Tamil Nadu, India

\begin{abstract}
Maintaining proper physical posture is essential for pain free life. This is more important while practicing exercise, sport and yoga asana. If not, miss-alignment of joints will lead to issues like fracture, muscle pain, stress on spine. Precise depictions of postures play a vital role while using assistive technology while practicing yoga. Maintenance of correct posture is in other words maintaining the Centre of Gravity (CoG) in correct location for that posture. By means of CoG, trainer module of assistive systems can identify the strain on any particular body part. By looking into CoG of consecutive postures, trainer can instruct corrections to maintain the postures. CoG value represents whether proper weight distribution is there among the body parts. This paper analyses the variation in CoG of various postures among the practitioners. Evaluation is done withbothmale and female yoga asana practitioners, half of them with prior practice. We identified that male practitioners have better CoGvaluesthan female, Similar is the observation for the practitioners with prior practice exposure also
\end{abstract}

KEYWORDS: Yoga, Time series, CoG, assisted learning, Joint Angles, Skeletal Information

Received: Apr 17, 2020; Accepted: May 07, 2020; Published: Jun 26, 2020; Paper Id.: IJMPERDJUN2020104

\section{INTRODUCTION}

Recognizing human activities from the associated video is one of the major research topic in the field of rehabilitation and assistive technology. This field finds its place in many applications such as machine learning, pattern recognition, human computer interaction [1-2]. The most useful rehabilitative methods include human activities like exercise and yoga practices. While doing yoga, maintaining perfect posture is mandatory failing which the practitioner may end up with injuries. Thus, accurate representations of postures play a vital role while using assistive technology. The assistive system should decide a particular posture as valid as per the human biomechanics. Modeling should consider the diverse nature of asana postures. Joint-angle limits are one of the accurate methods to evaluate the position of the connected joints as there is established biomechanical limits between any two pair of bone joints [3-4]. For instance, how much one can rotate the joint is based on the joint type. That is, we can rotate our shoulder joint to 360degreewhereas elbow joint cannotbefoldedbeyond180 degree. Limits of some of the joint-angles for some postures are defined in the books [5].

In order to define posture accurately, it is beneficial to use skeleton as connected segments. Every pair of consecutive joints of a bone is called as segment. Modeling human posture with assumption of either fixed joint- 
angle limits [7-9] or elbow and shoulder joint limits [10] alone may not be useful for applications such as asana training. In this paper, we are considering the biomechanical limits of hip, knee and ankle joints also, as studies in [11] shows that yoga helps in reduction of lower-back pain, and improves hip and spinal flexibility which in turn helps in reducing lowering back pain [12-13].

To maintain stability, the due considerations are on the factors such as (i) height of $\mathrm{CoG}$ (ii) the horizontal distance from the edge of the support of $\mathrm{CoG}$, (iii)the size of base of support, (iv) direction of the base in relation to the force (v) weight of the body. In this paper, we focus on CoG of every posture in asana sequence. Using CoG, trainer can identify the strain on any particular body part. Every asana consists of a sequence of poses. Transformation from one posture to another can be defined in the form of angle difference between the consecutive frames. Finding $\mathrm{CoG}$ of consecutive postures will enable the trainer to instruct corrections.

In this paper, we have taken Trigonasana to find CoG for 20 participants. Participants group is a mixture of male and female practitioners, consisting of 10 members in each category. Their age ranges from 20 to 50, average weight of females is $55.5 \mathrm{Kg}$ and that of male is $58.3 \mathrm{~kg}$. The average height of female is $155 \mathrm{~cm}$ and that of male is $170 \mathrm{~cm}$. Participants in the age group of $20-25$ have shown perfect postures, where as there is a variation in posture among the people in the age group, 45-50. Especially females and participants from both gender in the age group above 45 find it difficult to maintain this posture properly. To maintain balance, some participants shifted their hip and shoulders. It is also noticed that a lateral direction bend to maintain their centre of gravity. Their CoG will pass along the leg because of the bend.

In the rest of this paper, section II discusses related work on skeletal representation, section III briefs on directional planes and bio-mechanics of human body and section IV describes the calculations on finding centre of gravity for the postural sequence. Section V discusses the results and section VI concludes the paper.

\section{Related Work}

There are many techniques available to represent and recognize human activities. Symmetric motion patterns based on the centre [14] obtained from RGB and pair wise relative positions of joints extracted from depth frames are combined to recognize human actions.

Depth images [15]area sequence of features relying on Grassmann manifold to describe the dynamic of human body using its geometric appearance rather than using joint values. Depth and skeleton joint information can [16] represent the node of interest and to have improved recognition rate, depth information that help in extracting human silhouette to be used along with skeletal features.

In [17], A.Liu et. al., proposed coupled Hidden Conditional Model Fields (cHCRF) which combines graph-based methods of sequential modeling and visual feature sequences from depth data to recognize human activity.

In [18], authors proposed representationwith 15 skeleton joints called APJ3D. They found a set of keypostures by computing relative positions and local angles and have done classification using random forest. By considering human activity as a sequence of pose over time, by using 8 pairs of angles, they processed skeletal data to get invariant poses, followed by recognition phase, which uses the dissimilarity space of the representation. 
Relative geometry between body parts is more useful in activity recognition than their absolute locations. To classify human actions dictionaries of human parts using spatial and temporal joint coordinates [19] ora moving pose descriptor may be used. Similarly, body parts estimated from geometric relationships [20], or body joint angles estimated from a pair of stereo images[21] are used as well.

In computer graphics, many methods exists to represent human pose and its identification. To estimate the posture, Wei and Chai [22] and Lin et al.[23] used extra parameters such as difference between consecutive base support positioning, and distance between joints.

Studies such as [24], show that estimation of limb lengths is possible using statistical limits on their ratios. Similarly, by using body measurements, gender, and height, one can estimate the bone lengths[25].Signal processing techniques may be applied on skeletal information to classify human actions as proposed in [26].

Joint coordinates can differentiate human actions [26]. It is possible to design posture recognition and feedback system by using depth sensors like Microsoft Kinect which provides skeletal joint information. They are good sources as they are not affected by the light variations in the environment. Segmentation [27] of skeletal information is easier to do using this software. In some applications like self-assisted yoga practice, privacy is of prime concern of the practitioner. Since depth sensors can offer privacy preserving, the possibility of acquiring and representing human body skeleton from depth sensors ensures higher level of privacy[28]. In this paper, we used the joint coordinate values to find the postural stability. We represent the asana as a sequence of postures along with the joint differences of every successive posture and find Centre of Gravity of every step or posture in the asana.

\section{Biomechanics of human body}

Human skeleton has different types of joints; Figure 1 shows various types of joints in human body. Human can move their body in three orientations namely sagittal, transverse and coronal as shown in figure 2. Not all directional movements are possible in all the joints. For example, forward movement is possible in hip joints, where as it is not possible in knee joints. Similarly, the range of motions can also vary depending on the type of joints. 


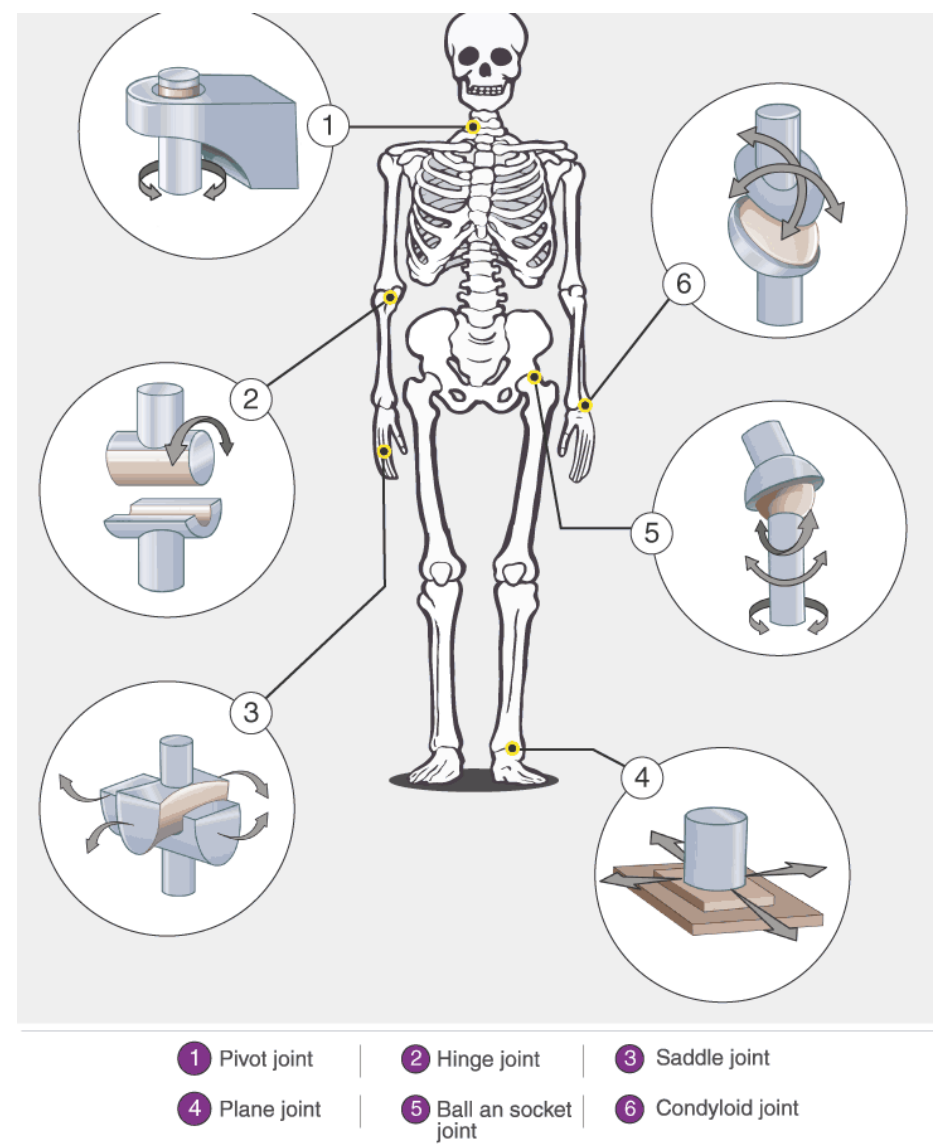

Figure 1: Types of Joints in Human Skeleton (Source: Https://Www.Byjus.Com).

Frontal
the frontal plane passes through
the body into anterior and
posterior portions.
The transverse plane passes
through the body in a line parallel
to the floor, dividing the body into
top and bottom portions.
Description
through the body from front to
and right portions.

Figure 2: Directional Planes and Movements of Human Skeleton (Source Https://Www.Ptdirect.Com/). 
Human can move or rotate their joints independent of other joints. It is possible to simultaneously move joints like hinge joint and pivot joint or ball and socket joints.

Rotation limits of joint angles vary based on the type of joints. Joints are the important regulatory mechanism of human body stability control. It is the process which precedes movements of human body parts to maintain balance in a changing environment. Centre of Gravity may influence the postural control and other morphological characters may influence the postural stability. Many studies show that there is a significant correlation between balance control and morphological property. For example, bearing weight on the right leg in the standing position depends on the morphological characters such as weight, height, lower limb length, upper limb length, width and length of foot.

Body weight is borne by skeletal system itself when human being are standing erect, where as in different standing position like 30 degree knee/hip flexion, 60 degree knee/hip flexion. Each leg will share certain percentage of body weight (right and left body weight).

Human spine muscles are responsible for keeping the head and trunk upright. Sufficient stiffness in the spinal is needed to bear the upper body weight. We can ensure this stiffness by many muscle group such as abdominal, lumborum muscles. To have a good and healthy spinal, core muscles should support the spine. The fact is that the muscles cannot be in contract position all the time. The muscles have to change their contractions and extensions by varying the weight bearing stress on the core.

When doing asanas, human being will stretch; contract the muscles that contribute to the postural changes. Studying the effect of asana postures on the human body part will help the trainer to give best advices. To find better postures, which will not do harm to human joints depends on the CoG of the particular posture and base support. The gravity force upon human structure has to be varied between the points of contact of human body with ground, so that no parts of the body will be under stress.

Generally, the point of contacts or otherwise called base support of human body vary based on the postures. Fig. 3 shows various base support while doing asanas.

In order to create assistive systems to correct miss-postural alignment for human being during sports and yoga practice, itis essential to know the details of joints positions. In this paper, we also used locations of joints to find $\mathrm{CoG}$, which help us to ensure that particular posture will not be harmful to muscles and joints.

From the skeletal information (i.e.) joint coordinate values, we extracted postural features. We represented joints details of human as a setoff joint coordinate values. By using Kinect software, we extracted joint coordinate values of human posture from the images captured by depth camera. Figure 4 shows the joints that can be tracked by Kinect software. 


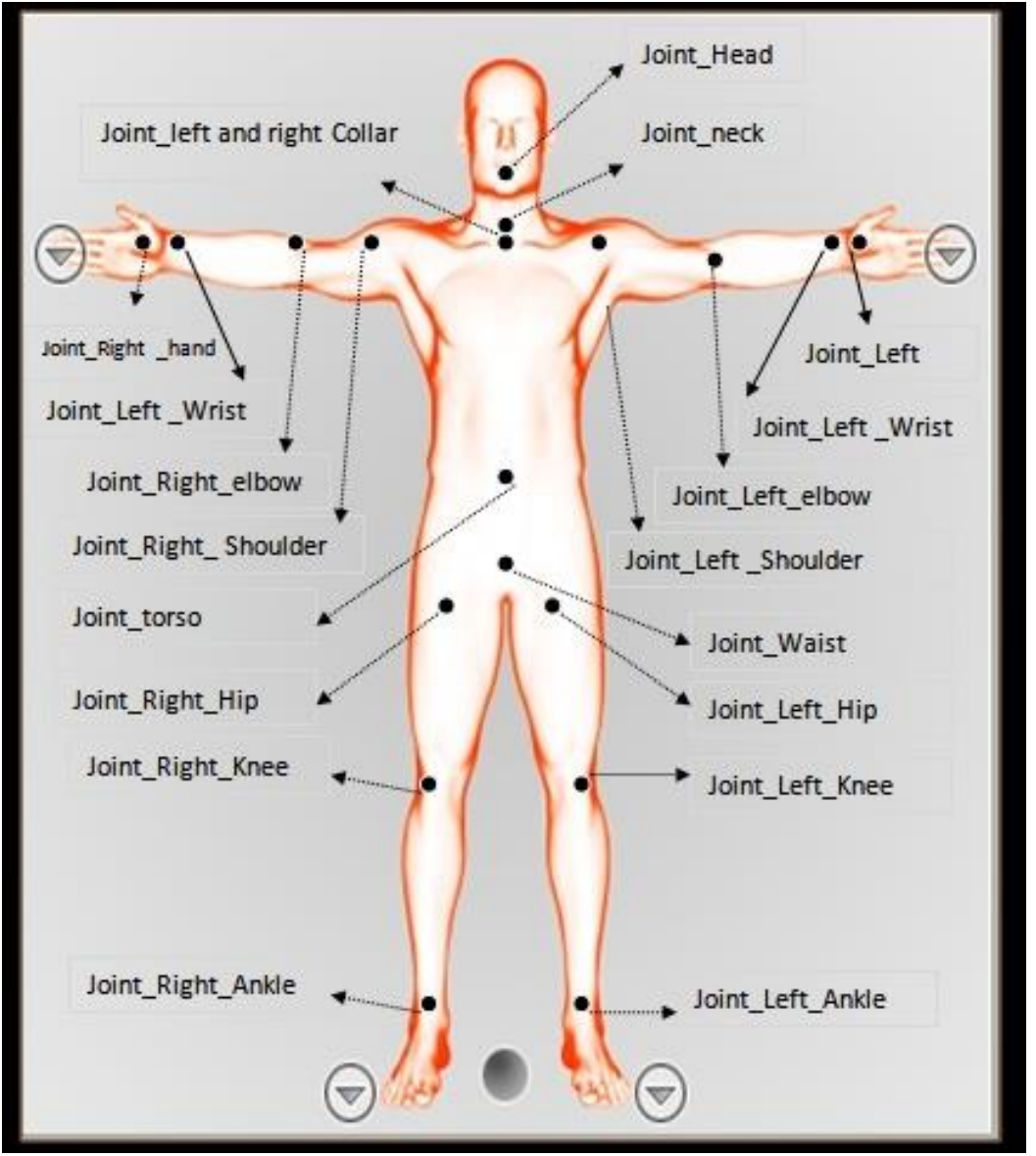

Figure 4: Skeletal Joints of Human Being.

Joint coordinate values thus extracted can be used to find the CoG of that posture. Following section discusses the proposed method of finding CoGs of every posture of a particular asana.

\section{Proposed Work}

Every asana involves a sequence of postures. Human being should be in a posture for a stipulated time while doing asana. We can redefine asana as a time series sequence of postures. At any particular point of time T0, the position of skeletal joint scan define a posture. We can define an asana as a set of postures as follows

Asana $=\{($ Posture_1 $),($ Posture_2,D1 $), . .($ Posture_n, Dn $)\}$

where D1 represents the time duration of the posture, and posture_1 is a matrix of $\mathrm{x}, \mathrm{y}$ coordinates of joints. Now, we can represent a posture as

$$
\text { Posture_I }=\left[J_{i 1}, J_{i 2}, \ldots J_{i p}\right]
$$

where J's are points with $\mathrm{x}$ and $\mathrm{y}$ coordinate values of posture $\mathrm{i}$ in the asana.

Let, Diff be the difference in the joint angle from postureI-1 to posture I represented as follows.

$$
\text { Diff_I-1,I }=\left[r_{i j 1}, r_{i j 2}, \ldots r_{i j n}\right]
$$

Every yoga asana consists of different poses. But variation from one posture to the other will involve 
only certain number of joints. For example, in Chaturangadandasana (four-limbed staff pose), the difference between consecutive steps is only in the position of elbow joints as explained in Figure 5 and Figure 6. The flow of work is shown in the Figure 7. Algorithm 1 is used to calculate the difference matrix.

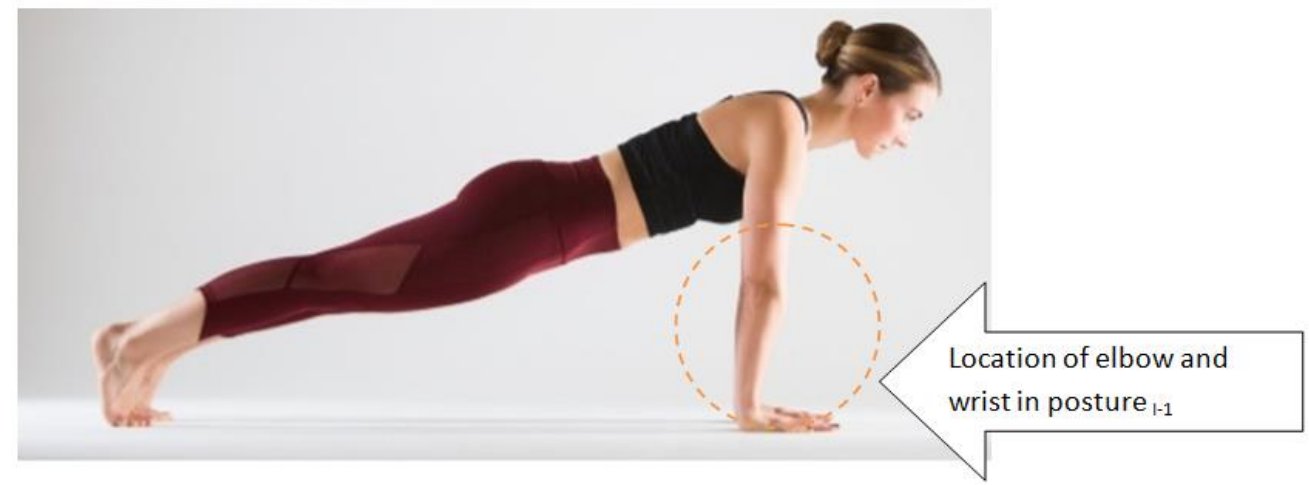

Figure 5: Pose (I-1) of Chaturangadandasana

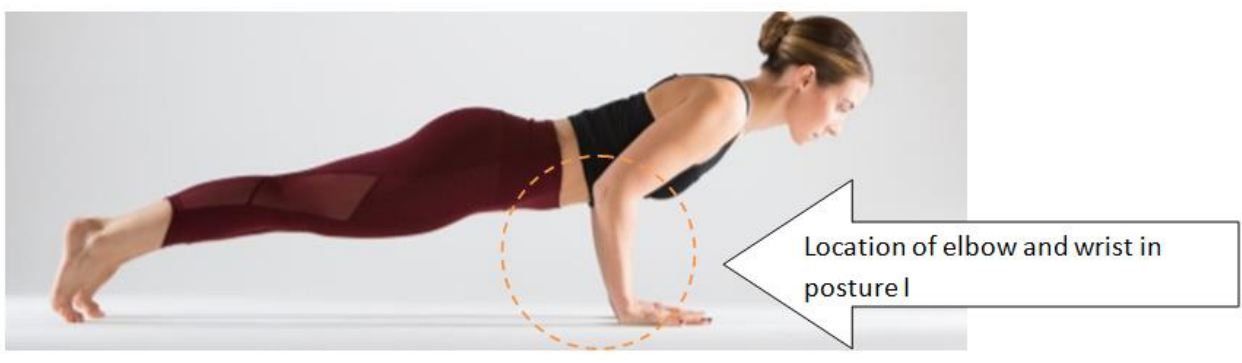

Figure 6: Pose (I) ofChaturangadandasana

Transformation matrix can represent the location change in joint wrist (both left and right) propagated to joint elbow (both left and right). People who practice asana should remain in same asana posture for a certain amount of time like two to five seconds and some times more than that also. Time information needs to be stored in the data base along with the postural description so that assistive system can identify the number of frames to be skipped for next postural calculation.

Once the frame for which we need to calculate the CoG identified, we can extract the coordinate value of the joints. In order to find $\mathrm{CoG}$ of a posture, we can use the terms proximal and distal to represent the distance of body parts from the centre of the body. A joint of a segment is proximal if it closer to reference point. Distal joint is the one which is further away from reference point. For example, considering the torso as reference point, shoulder is proximal and elbow is distal. Algorithm 2 shows the steps needed to calculate CoG.

Using Table 1, we can find the Centre of Mass of a segment by using the segment's proximal and distal joints. 
Table 1: Segment Length Proportions

\begin{tabular}{|l|c|c|}
\hline \multirow{2}{*}{\multicolumn{1}{|c|}{ Segment }} & \multicolumn{2}{c|}{ Centre of Gravity (\% Length) } \\
\cline { 2 - 3 } & Female & Male \\
\hline Head \& Neck & 48.41 & 50.02 \\
\hline Trunk & 37.82 & 43.10 \\
\hline Upper Arm(both left \& right) & 57.54 & 57.72 \\
\hline Fore Arm(both left \& right) & 5.59 & 5.74 \\
\hline Hand & 74.74 & 8.00 \\
\hline Thigh & 36.12 & 40.95 \\
\hline Shank & 43.52 & 3.95 \\
\hline Foot & 4.14 & 4.15 \\
\hline
\end{tabular}

Human body has the symmetric nature. It is convenient to use the percentage length of right forearm for left forearm also. The same concept is applicable to upper arm, hand, thigh, lower leg and foot also.

Table 2: Proximal and Distal Points of Segments

\begin{tabular}{|l|l|l|}
\hline \multicolumn{1}{|c|}{ Segment } & \multicolumn{1}{c|}{ Proximal } & \multicolumn{1}{c|}{ Distal } \\
\hline Head \&Neck & Head & Neck \\
\hline Trunk & Centre of shoulder joints & Centre of Hip joints \\
\hline Upper Arm(s) & Shoulder joint & Elbow joint \\
\hline Fore Arm(s) & Elbow joint & Wrist joint \\
\hline Hand & Wrist Joint & Finger Tip \\
\hline Thigh & Hip joint & knee joint \\
\hline Shank & Knee joint & Ankle joint \\
\hline Foot & Ankle & Toe \\
\hline
\end{tabular}

From the skeletal values, we can assign one end of the segment proximal and the other end as distal. Table 2 shows the joint points of every segment.

Eq.(1) helps to calculate CoG of X coordinate of a segment and similarly Eq.92) helps to calculate CoG of $\mathrm{Y}$ coordinate of a segment.

Based on these values, Eq.(3) and Eq.(4) can help to calculate $\mathrm{X}$ and $\mathrm{Y}$ coordinate value $\mathrm{CoG}$ of the whole body.

$$
\begin{aligned}
& x \operatorname{cog}_{\text {segmenti }}=\left(x \text { distal } l_{\text {segment }}-x \text { proximal }{ }_{\text {segment }}\right) *(\% \mathrm{~cm} \text { of segment }) \\
& + \text { xproximal }_{\text {segment }}(1) \\
& \text { ycog }{ }_{\text {segmenti }}=\left(\text { ydistal } \text { segment }- \text { yproximal }_{\text {segment }}\right) *(\% \mathrm{~cm} \text { of segment }) \\
& + \text { yproximal }_{\text {segment }}(2) \\
& x \text { com }_{\text {wholebody }}=\frac{\sum_{i} m i * x \operatorname{cog}_{\text {segmenti }}}{\sum_{i} m_{i}} \\
& \text { ycom }_{\text {wholebody }}=\frac{\sum_{i} m i * y \operatorname{cog}_{\text {segmenti }}}{\sum_{i} m_{i}}
\end{aligned}
$$




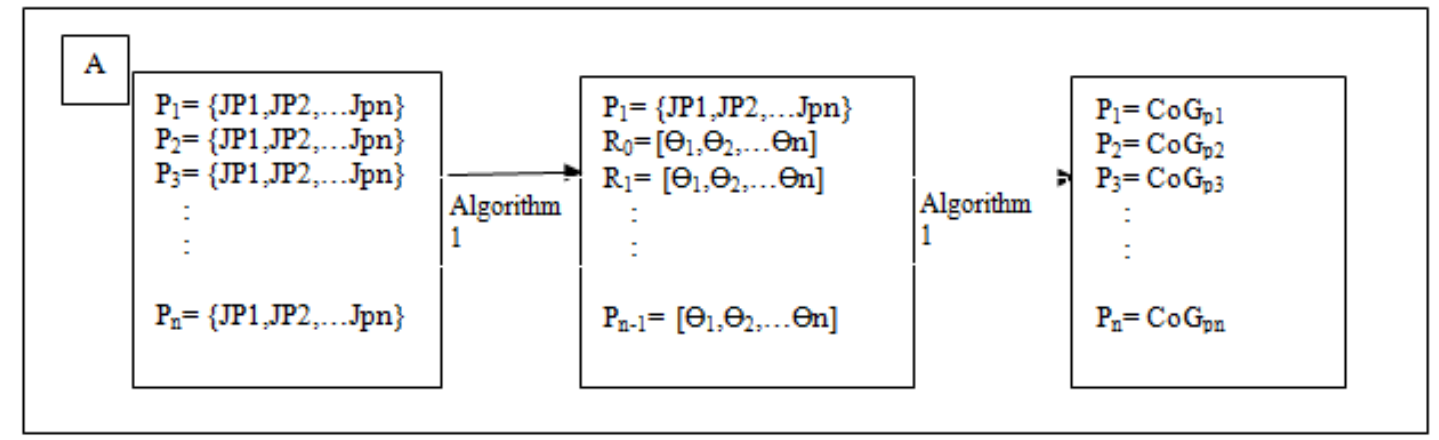

Figure 7: Flow of Work.

\section{Algorithm 1: To Find Difference Matrix}

Input:

- $\operatorname{Set} A=\{P 1, P 2, \ldots P n\} ; P_{1}$ is the first posture in asana $A, \ldots, P_{n}$ is the last posture of the asana.

- $\quad P_{i}=\left\{H P_{i}\right\}$, Joint angle matrix

Output: $n$ number of $R_{i}$, difference matrices for the joints corresponding to every posture in the asana

\section{Begin}

$\mathrm{i}=2$

For each HP in A

For each JP in $\{\mathrm{HP}\}$

$$
\mathrm{R}_{\mathrm{i}}=\mathrm{JP}_{\mathrm{i}}-\mathrm{JP}_{\mathrm{i}-1}
$$

End loop

End loop

\section{End}

Algorithm 2: for finding COG of a posture

Input:

- $H P i ; i=1 . . n$

- $\quad$ Table for segmental length proportion

Output: Array $\mathrm{CoG}_{i}$, Center of Gravity for pose $\mathrm{HP}_{i} ; i=1$ to $n$

\section{Begin}

For every posture of the asana

For each segment in segment array

find the proximal and distal of the segment $\mathrm{i}_{\mathrm{i}}$ 
find the CoG using Eq.(1-4)

store it in $\mathrm{CoG}_{\mathrm{i}}$

End Loop

End Loop

End

\section{Experimental Results}

Evaluation is done using the available yoga data set in[30]. We made actors to perform every asana two times and then calculated $\mathrm{CoG}$ for certain postures of performed asana sequence and then compared the calculated CoG with the one available in database for the corresponding asana. Our proposed algorithms took the users asana performance and calculated data segment values and also distal and proximal values of each segment.

We considered the female practitioners separately for $\mathrm{CoG}$ calculations and comparisons. Though there is no distinction in the data set about male and female data, we compared our observations separately with the data set. Female practitioners showed variations from their first time practice and the second time practice as shown in figure 8 .

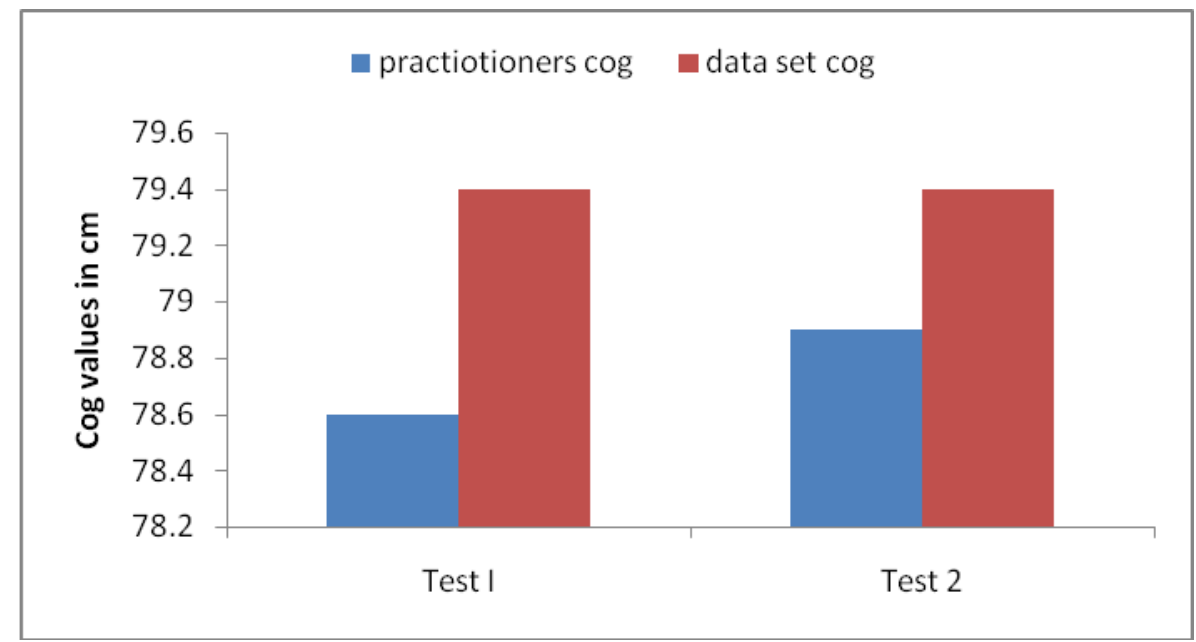

Figure 8: Comparison of Cog Values of Female Practitioners.

Similar is the observation with the practitioners of age above forty who found it difficult to maintain the balance as shown in figure 9. 


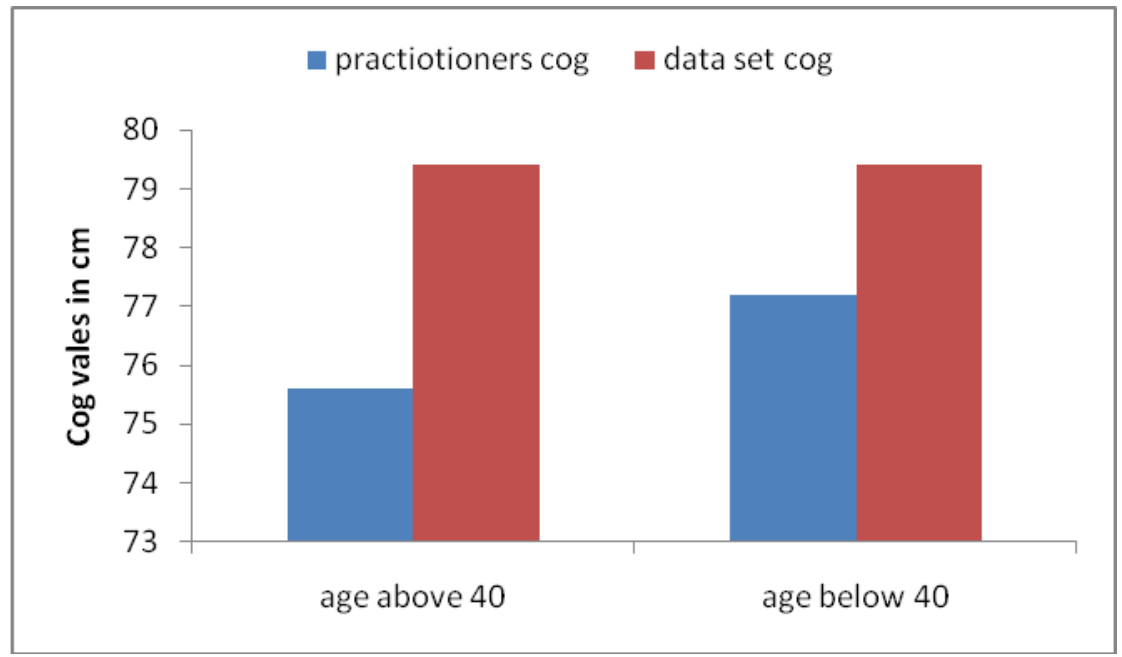

Figure 9: Effect of Age in COG.

Practitioners with prior practice showed a closer CoG value compared to the dataset as shown in Figure 10.

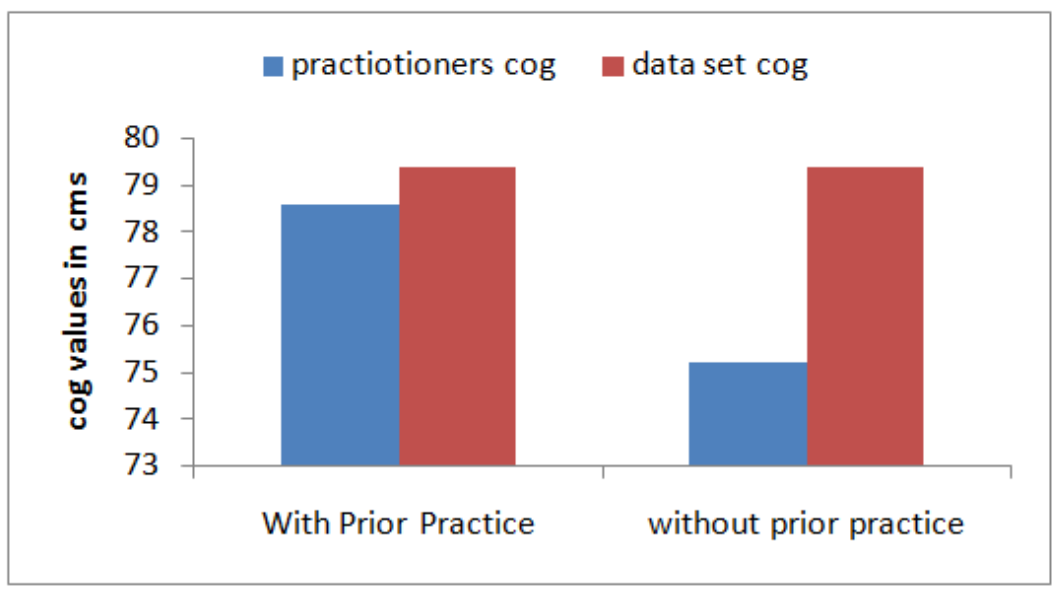

Fig.10: Effect of practice in $\mathrm{CoG}$

Female and male practitioners CoG values for the two test average is compared in Figure 11. Male practitioners showed better consistent performance. 


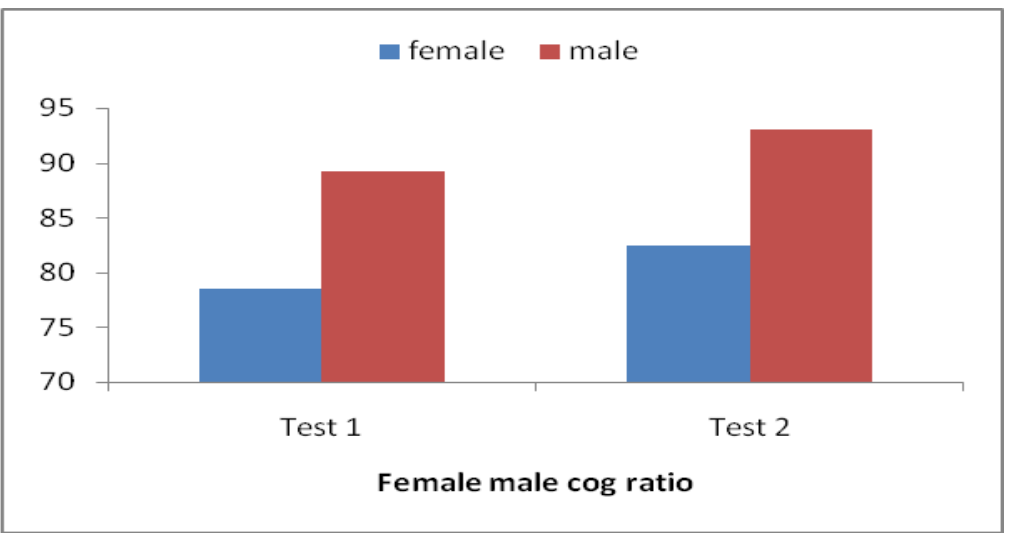

Figure 11: Male and Female Practitioners Cog Variations.

\section{CONCLUSIONS}

Performing yoga with correct postures is essential to maintain stability. The CoG value represents whether proper weight distribution is there among the body parts. This paper shows that CoG of postures may vary and cannot have the exact value by all practitioners. So, being in correct posture may vary from person to person. In such cases, other morphological factors may help in maintaining proper weight distribution. The factors that influence the weight distribution have to be further studied along with $\mathrm{CoG}$, so that trainers can offer personalized corrections to every individual while we design assistive systems. In future, we plan to consider other morphological properties that influence the postures for weight distribution in every posture to avoid injuries.

\section{REFERENCES}

1. P. Turaga, R. Chellapa, V. S. Subrahmanian, and O. Udrea. Machine recognition of human activities: A survey. IEEE Transactions on Circuits and Systems for Video Technology, 18(11):1473-1488, 2008.

2. J. K. Aggarwal and M. S. Ryoo. Human activity analysis: A review. In ACM Computing Surveys, 2011.

3. H. Hatze. A three-dimensional multivariate model of passive human joint torques and articular boundaries. Clinical Biomechanics, 12(2):128-135, 1997.

4. T. Kodek and M. Munich. Identifying shoulder and elbow passive moments and muscle contributions. In IEEE Int.Conf.onIntelligentRobotsandSystems, volume2,pages 1391-1396, 2002.

5. U. S. N. Aeronautics and S. Administration. NASA-STD3000: Man-systems integration standards. Number v. 3 in NASA-STD. National Aeronautics and Space Administration, 1995.

6. Rathod, C. H. A. N. D. A. R., and GONDI KONDA Reddy. "Experimental investigation of angular distortion and transverse shrinkage in $\mathrm{CO} 2$ arc welding process." International Journal of Mechanical Engineering, 5 (4), 2128 (2016).

7. https://www.reddit.com/r/datasets/search?q=yoga

8. J. Chen, S. Nie, and Q. Ji. Data-free prior model for upper bodyposeestimationandtracking, IEEETrans.ImageProc., 22(12):4627-4639, Dec. 2013.

9. J. M. Rehg, D. D. Morris, and T. Kanade. Ambiguities in visual tracking of articulated objects using twoand threedimensional models. The International Journal of Robotics Research, 22(6):393-418, 2003. 
10. C. Sminchisescu and B. Triggs. Estimating articulated human motion with covariance scaled sampling. The International Journal of Robotics Research, 22(6):371-391, 2003.

11. Ismail, Mahmud Rasheed, Muhannad Al-Waily, and Ameer A. Kadhim. "Biomechanical Analysis and Gait Assessment for Normal and Braced Legs." International Journal of Mechanical \& Mechatronics Engineering IJMME-IJENS 18.03 (2018).

12. L. Herda, R. Urtasun, and P. Fua. Hierarchical implicit surface joint limits for human body tracking. Computer Vision and Image Understanding, 99(2):189-209, 2005.

13. Pittala, Suresh, and T. Diriba. "Computational Fluid Dynamics Analysis of Impeller Design For A Pump." International Journal of Mechanical Engineering (IJME) 5.4.

14. Cramer H, Lauche R, Haller H, Dobos $G$. A systematic review and meta-analysis of yoga for low back pain. Clin J Pain. 2013;29:450-60.

15. Williams K, Abildso C, Steinberg L, Doyle E, Epstein B, Smith D, et al. Evaluation of the effectiveness and efficacy of Iyengar yoga therapy on chronic low back pain. Spine (Phila Pa 1976) 2009;34:2066-76.

16. D.Galantino ML, Bzdewka TM, Eissler-Russo JL, Holbrook ML, Mogck EP, Geigle P, et al. The impact of modified Hatha yoga on chronic low back pain: A pilot study. AlternTher Health Med. 2004;10:56-9.

17. Deshpande, Vivek, and P. M. George. "Kinematic Modelling and Analysis of 5 DOF Robotic Arm." International Journal of Robotics Research and Development (IJRRD) 4.2 (2014): 17-24.

18. J.Luo,W.Wang, andH.Qi, "Spatio-temporalfeatureextraction and representation for RGB-D human action recognition," PatternRecognitionLetters, vol.50,pp.139-148,2014.

19. R. Slama, H. Wannous, and M. Daoudi, "Grassmannian representation of motion depth for $3 D$ human gesture and action recognition, "inProceedingsofthe22ndInternationalConference onPatternRecognition(ICPR'14),pp.3499-3504,IEEE,Stockholm,Sweden,August2014.

20. Ismail, Mahmud Rasheed, Muhannad Al-Waily, and Ameer A. Kadhim. "Biomechanical Analysis and Gait Assessment for Normal and Braced Legs." International Journal of Mechanical \& Mechatronics Engineering IJMME-IJENS 18.03 (2018).

21. G. Chen, D. Clarke, M. Giuliani, A. Gaschler, and A. Knoll, "Combining unsupervised learning and discrimination for 3D actionrecognition, "SignalProcessing,vol.110,pp.67-81,2015.

22. A.-A. Liu, W.-Z. Nie, Y.-T. Su, L. Ma, T. Hao, and Z.-X. Yang, "CoupledhiddenconditionalrandomfieldsforR $G B$ Dhumanactionrecognition, "SignalProcessing,vol.112,pp.74-82,2015.

23. J.Wang,Z.Liu,Y.Wu,andJ.Yuan, “Miningactionletensembleforactionrecognitionwithdepthcameras, ”inPro ceedingsofthe IEEE Conference on Computer Vision and Pattern Recognition (CVPR'12),pp.12901297,Providence,RI,USA,June2012.

24. Shukla, D. K., M. A. H. E. N. D. R. A. Singh, and K. K. Jain. "Bearing Capacity Of Footing On Slopping Anisotropic Rock Mass." IMPACT: International Journal of Research in Engineering \& Technology 
(2014).

25. C. Wang, Y. Wang, A. L. Yuille, Mining 3d key-pose-motifs for action recognition, in: Proceedings of the IEEE Conference on Computer Vision and Pattern Recognition (CVPR), 2016, pp. 2639-2647.

26. R. Vemulapalli, F. Arrate, and R. Chellappa. Human action recognition by representing $3 d$ skeletons as points in a lie group. In IEEE Conference on Computer Vision and Pattern Recognition, pages 588-595. IEEE, 2014

27. M. Z. Uddin, N. D. Thang, J.T. Kim and T.S. Kim, Human Activity Recognition Using Body Joint-Angle Features and Hidden Markov Model. ETRI Journal, vol.33, no.4, Aug., pp.569-579, 2011

28. X. K. Wei and J. Chai. Intuitive interactive human-character posing with millions of example poses. Computer Graphics and Applications, IEEE, 31(4):78-88, 2011.

29. J. Lin, T. Igarashi, J. Mitani, M. Liao, and Y. He. A sketching interface for sitting pose design in the virtual environment. Visualization and Computer Graphics, IEEE Transactions on, 18(11):1979-1991, 2012.

30. C. BenAbdelkader and Y. Yacoob. Statistical estimation of human anthropometry from a single uncalibrated image. In Methods, Applications, and Challenges in Computer assisted Criminal Investigations, Studies in Computational Intelligence. Springer-Verlag, 2008.

31. P. Guan, A. Weiss, A. Balan, and M. J. Black. Estimating human shape and pose from a single image. In Int. Conf. on Computer Vision, ICCV, pages 1381-1388, Sept. 2009

32. T. Batabyal, A. Vaccari, S. T. Acton, Ugrasp: A unified framework for activity recognition and person identification using graph signal processing, in: 2015 IEEE International Conference on Image Processing (ICIP), IEEE, 2015, pp. 3270-3274.

33. S. Gasparrini, E. Cippitelli, E. Gambi, S. Spinsante, and F. Fl'orez-Revuelta, "Performance analysis of self-organisingneural networks tracking algorithms for intake monitoring using kinect, " in Proceedings of the 1st IET International Conference on Technologies for Active and Assisted Living (TechAAL '15), Kingston, UK,November2015.

34. J. R. Padilla-L'opez, A. A. Chaaraoui, F. Gu, and F. Fl'orezRevuelta, "Visual privacy by context: proposal and evaluation ofalevel-basedvisualisationscheme,"Sensors,vol.15,no.6,pp. 1295912982,2015 .

35. Ponmozhi.K, Deepalakshmi. P, 2018. Posture recognition system for Assisted self-learning of yoga by Cognitive impaired older People for the prevention of Falls, Int. Conf. on Big Data Innovation for Sustainable Cognitive Computing, Coimbatore, India, 13-15 Dec. 2018.

36. https://www.reddit.com/r/datasets/search?q=yoga 